Mon. Not. R. Astron. Soc. 000,1] (2017) Printed 3 April $2018 \quad$ (MN LATEX style file v2.2)

\title{
Further Observational Evidence for a Critical Ionising Luminosity in Active Galaxies
}

\author{
S. J. Curran ${ }^{1 \star}$, R. W. Hunstead ${ }^{2}$, H. M. Johnston ${ }^{2}$, M. T. Whiting ${ }^{3}$, E. M. Sadler ${ }^{2}$, \\ J. R. Allison ${ }^{3}$ and C. Bignell ${ }^{4}$ \\ ${ }^{1}$ School of Chemical and Physical Sciences, Victoria University of Wellington, PO Box 600, Wellington 6140, New Zealand \\ ${ }^{2}$ Sydney Institute for Astronomy, School of Physics, The University of Sydney, NSW 2006, Australia \\ ${ }^{3}$ CSIRO Astronomy and Space Science, PO Box 76, Epping NSW 1710, Australia \\ ${ }^{4}$ National Radio Astronomy Observatory, P.O. Box 2, Rt. 28/92 Green Bank, WV 24944-0002, USA
}

Accepted -. Received —; in original form -

\begin{abstract}
We report the results of a survey for H I 21-cm absorption at redshifts of $z \gtrsim 2.6$ in a new sample of radio sources with the Green Bank and Giant Metrewave Radio Telescopes. From a total of 25 targets, we report zero detections in the 16 for which optical depth limits could be obtained. Based upon the detection rate for $z \geqslant 0.1$ associated absorption, we would expect approximately four detections. Of the 11 which have previously not been searched, there is sufficient source-frame optical/ultra-violet photometry to determine the ionising photon rate for four. Adding these to the literature, the hypothesis that there is a critical rate of $Q_{\mathrm{HI}} \sim 10^{56}$ ionising photons per second (a monochromatic $\lambda=912 \AA$ luminosity of $L_{\mathrm{UV}} \sim 10^{23} \mathrm{~W} \mathrm{~Hz}^{-1}$ ) is now significant at $\approx 7 \sigma$. This reaffirms our assertion that searching $z \gtrsim 3$ active galaxies for which optical redshifts are available selects sources in which the ultra-violet luminosity is sufficient to ionise all of the neutral gas in the host galaxy.
\end{abstract}

Key words: galaxies: active - quasars: absorption lines - radio lines: galaxies - ultra violet: galaxies - galaxies: fundamental parameters - galaxies: ISM

\section{INTRODUCTION}

While absorption of the 21-cm spin-flip transition of neutral hydrogen $(\mathrm{HI})$ is readily detected in near-by galaxies, it is conspicuous by its absence from the redshifted Universe. H I 21-cm absorption traces the cool component of the gas, the reservoir for star forma' tion, which can be detected in either quiescent galaxies intervening the line-of-sight to a more distant radio source or associated with the host galaxy of the radio source itself. While the detection of hydrogen at radio wavelengths is not susceptible to the dust extinction effects of optical/ultra-violet band searches (Carilli et al. 1998; Curran et al. 2006, 2017), after over three decades of searching (e.g. Davis \& May 1978; de Waard et al. 1985) there are only 50 detections each of $\mathrm{HI} 21-\mathrm{cm}$ at $z \gtrsim 0.1$ in either intervening or associated absorption (compiled in Curran et al. 2016b). This is despite $\mathrm{HI}$ being detected through Lyman- $\alpha$ absorption in 12000 damped Lyman- $\alpha$ absorbers (DLAs) and sub-DLAs 1 (Noterdaeme et al. 2012), mainly at $z \gtrsim 1.7$ where the Lyman- $\alpha$

\footnotetext{
* Stephen.Curran@vuw.ac.nz

1 A DLA is defined as having a neutral hydrogen column density exceeding $N_{\mathrm{HI}}=2 \times 10^{20}$ atoms $\mathrm{cm}^{-2}$, with sub-DLAs having lower column densities, although exhibiting the same characteristic damping wings in the absorption profile.
}

transition is redshifted into the atmospheric observing window 2 By comparison, the detection of $\mathrm{H}$ I $21-\mathrm{cm}$ absorption occurs overwhelmingly at redshifts of $z \lesssim 1$, or less than half the look-back time to the origin of the Universe. While much of this can be attributed to the past availability of the suitable radio bands, detection rates at $z \gtrsim 1$ are demonstrably lower than at $z \lesssim 1$ Kanekar \& Chengalur 2003; Curran et al. 2013c).

For the intervening absorbers it has been argued that this is due to an evolution in the spin temperature, $T_{\mathrm{s}}$, of the gas, where the fraction of the cold neutral medium (CNM, where $T \sim 150 \mathrm{~K}$ and $n \sim 10 \mathrm{~cm}^{-3}$ ) decreases with redshift Kanekar \& Chengalur 2003; Kanekar et al. 2014). However, given that the comparison of the 21-cm absorption strength with the total neutral hydrogen column density, from the Lyman- $\alpha$ transition, can at best yield the spin temperature/covering factor degeneracy $\left(T_{\mathrm{s}} / f\right)$, Curran et al. (2005) argued that an apparent evolution in $T_{\mathrm{S}}$ could be caused by the assumption that $f=1$ for the high redshift absorbers. Furthermore, by accounting for the effects of an expanding Universe, a standard $\Lambda$ cosmology means that at redshifts of $z \gtrsim 1$ the absorber is always at a similar angular diameter distance as the background

\footnotetext{
2 At lower redshifts, a high $\mathrm{HI}$ column density is inferred from the equivalent width of the $\mathrm{Mg}$ II absorption and other metal ion transitions which can be detected by ground-based telescopes (e.g. Rao et al. 2006).
} 
continuum source, i.e. $D A_{\mathrm{abs}} \approx D A_{\mathrm{QSO}}$. In contrast, at $z \lesssim 1$ $D A_{\text {abs }}<D A_{\mathrm{QSO}}$ is possible (when $z_{\text {abs }}<z_{\mathrm{QSO}}$ ), suggesting that the mix of "spin temperatures" at $z \lesssim 1$ and exclusively high values at $z \gtrsim 1$ (Kanekar \& Chengalur 2003), is in fact the effect of geometry on the covering factor (Curran \& Webb 2006; Curran 2012). By deconstructing the covering factor, via the angular diameter distances and high resolution imaging of the background sources, there is now compelling evidence that the fraction of the $\mathrm{CNM}$ in intervening absorbers may trace the star formation history of the Universe (Curran 2017).

For the associated absorbers, since, by definition, $z_{\text {abs }} \approx$ $z_{\mathrm{QSO}}$, the same geometric effects cannot account for the difference in the detection rates between the low and high redshift sources. From a survey of H I 21-cm absorption at $z \gtrsim 3$, Curran et al. (2008) suggested that their exclusively non-detections were due to the high redshifts selecting sources with high rest-frame ultra-violet luminosities ( $L_{\mathrm{UV}} \gtrsim 10^{23} \mathrm{~W} \mathrm{~Hz}^{-1}$ ). Since an examination of the literature showed that H I 21-cm had never been detected at these luminosities, irrespective of redshift, they suggested that the nondetection of cool neutral gas was due to the high redshift selection yielding only sources where the UV luminosity was sufficient to excite the gas to below the detection limit. This observational result has since been confirmed several times for various heterogeneous samples Curran et al. 2011a, 2013b. c, 2016a, 2017; Allison et al. 2012; Geréb et al. 2015; Aditya et al. 2016; Grasha et al. 2017)3, showing this to be an ubiquitous effect.

In order to investigate why there is an apparent critical UV luminosity and why this is $L_{\mathrm{UV}} \sim 10^{23} \mathrm{~W} \mathrm{~Hz}^{-1}$, Curran \& Whiting (2012) applied the equation of photoionsation equilibrium (Osterbrock 1989) to a gas disk with an exponential density distribution (Begeman et al. 1991) and found that an ionising photon rate of $Q_{\mathrm{H}_{\mathrm{I}}} \equiv \int_{\nu}^{\infty}\left(L_{\nu} / h \nu\right) d \nu \approx 3 \times 10^{56} \mathrm{~s}^{-1}$ (a monochromatic $\lambda=912 \AA$ luminosity of $L_{\mathrm{UV}} \sim 10^{23} \mathrm{~W}$ $\mathrm{Hz}^{-1}$ ) will ionise a gas disk with the same scale-length as the Milky Way (Kalberla et al. 2007). This suggests that the observed critical luminosity is sufficient to ionise all of the neutral gas in a large spiral galaxy, thus explaining why $\mathrm{H}$ I $21-\mathrm{cm}$ has never been detected where $L_{\mathrm{UV}} \gtrsim 10^{23} \mathrm{~W} \mathrm{~Hz}^{-1}$. Since the vast majority of $z \gtrsim 1$ radio sources for which redshifts are available are believed to have luminosities above the critical value (see figure 4 of Morganti et al. 2015), this would mean that even the Square Kilometre Array (SKA) will be unable to detect H I 21-cm absorption in these objects.

Given this critical luminosity above which all of the gas in the host is ionised, in order to detect the cool, star-forming gas within high redshift radio sources, we need to dispense with the reliance upon an optical redshift, thus avoiding the selection of the most UV luminous objects. Through their wide instantaneous bandwidths, the SKA pathfinders are ideally suited to spanning a large range of redshift space in a single tuning (e.g. Allison et al. 2015; Maccagni et al. 2017) 4 However, these are generally limited to frequencies of $\gtrsim 700 \mathrm{MHz}$, i.e. H I 21 -cm at redshifts of $z \lesssim 1$, therefore requiring the SKA to find the missing cold neutral gas at high redshift. In the meantime, we can continue to search for H I 21-cm

3 Grasha et al. (2017), which is still in submission, reports 0 new detections of H I 21 -cm absorption out of 89 new searches over $0.02<z<3.8$ (see Grasha \& Darling 2011).

${ }^{4}$ Since the nature of the absorber is usually determined from an optical spectrum, other techniques for distinguishing intervening from associated absorption must be explored, with machine learning showing some promise Curran et al.2016b). absorption, via various search strategies, with currently available instruments (e.g. Curran et al. 2016a). From newly obtained redshifts for a sample of strong flat spectrum radio sources (see Sect. 2.1, we can perform a large survey for associated 21-cm absorption in active galaxies over redshift ranges spanning most of the Universe's history. Here we present the results of our high redshift $(z \gtrsim 2.6)$ survey with the Green Bank Telescope (GBT) and the Giant Metrewave Radio Telescope (GMRT).

\section{OBSERVATIONS AND ANALYSIS}

\subsection{The sample}

The Second Realization of the International Celestial Reference Frame by Very Long Baseline Interferometry (ICRF2, Ma et al. 2009), constitutes a sample of strong flat spectrum radio sources, of which 1682 now have known redshifts (Titov \& Malkin 2009; Titov et al. 2013 and references therein), yielding a tuning frequency in the search for 21-cm absorption. Being VLBI sources, all have significant compact flux, thus maximising the chance of a high covering factor and thus optical depth (Curran et al. 2013a). The original aim of the survey was to form part of a large observing campaign to search and quantify the incidence of associated H I 21 $\mathrm{cm}$ absorption over all redshifts, although observing time was only granted for the high redshift $(z \gtrsim 2.6$ ) proposals. As mentioned above (Sect.11, we believe that such a high redshift selection will yield only sources above the critical UV luminosity, where the detection of $\mathrm{HI} 21-\mathrm{cm}$ is unlikely. However, having a preconceived suspicion of the outcome should not preclude it from being tested.

\subsection{Observations and data reduction}

\subsubsection{GBT observations}

In the ICRF2, 1456 of the sources have declinations accessible to the GBT, in addition to being at a redshift which places the 21$\mathrm{cm}$ transition into a GBT band. For this, the high redshift survey, we used the PF1 290-395 MHz receiver (H I 21-cm over $z=2.6-3.9$ ). We formed our target list by prioritising sources with flux densities estimated to be in excess of $1 \mathrm{Jy}$ at the redshifted 21-cm frequency. Excluding the two which already had published searches at the time, left 24 targets. The observations were performed over 24-26 February 2014 with each source observed for a total of 1.3 hours in two orthogonal linear polarisations ( $X X \& Y Y$ ). The Prime Focus 1 (PF1) receiver was used, backed by the GBT spectrometer, with a bandwidth of $12.5 \mathrm{MHz}$, in order to minimise RFI while maintaining a velocity coverage of $\Delta v \approx \pm 5500 \mathrm{~km}$ $\mathrm{s}^{-1}$. A channel width of $11 \mathrm{kHz}$ gave a spectral resolution of $\approx 10 \mathrm{~km} \mathrm{~s}^{-1}$. The data were analysed, flagged for RFI and averaged using the GBTIDL software.

\subsubsection{GMRT observations}

In the ICRF2, 416 of the sources have declinations accessible to the GMRT, of which 54 have $21-\mathrm{cm}$ shifted to within the $90-\mathrm{cm}$ band (305-360 MHz, H I 21-cm over $z=2.9-3.7$ ). We prioritised sources with flux densities estimated to be in excess of $0.5 \mathrm{Jy}$ at the redshifted $21-\mathrm{cm}$ frequency. As per the GBT observations, the flux limit was chosen in order to attain high sensitivities, while still retaining a sizable number of targets. If the radio luminosity is correlated with the UV luminosity, it is conceivable that this selection may bias against the detection of cool, neutral gas. Although 
there is a correlation (Curran \& Whiting 2010), there is no critical rest-frame $1.4 \mathrm{GHz}$ luminosity (Curran et al. 2008), with the relationship probably arising from both luminosities being correlated with the redshift.

Excluding the potential targets with published searches for 21$\mathrm{cm}$ absorption at the time left 16 targets. The observations were taken with the full 30 antenna array over 9-13 November 2013 and 28-29 March 2014, with each source observed for a total of two hours in two orthogonal circular polarisations ( $L L \& R R)$. For bandpass calibration 3C 48, 3C 147 and 3C 298 were used, with the phases being self calibrated apart from $1427+543,1427+543$, 0521-262 \& 2318-087, which used a strong near-by unresolved source. For the backend we used the FX correlator over a bandwidth of $4 \mathrm{MHz}$, which over 512 channels gave a spectral resolution of $\approx 7 \mathrm{~km} \mathrm{~s}^{-1}$. The data were calibrated and flagged using the MIRIAD interferometry reduction package. After averaging the two polarisations, a spectrum was extracted from the cube. None of the sources was resolved by the synthesised beam, which ranged from $10.7^{\prime \prime} \times 9.4^{\prime \prime}$ to $30.2^{\prime \prime} \times 11.1^{\prime \prime}$.

\section{RESULTS}

\subsection{Observational results}

Of the 29 spectra, three were completely dominated by RFI and a further three had close to zero flux. In Fig. 1 we show the remaining 23 spectra, from 19 different targets. Of these, seven have $\mathrm{RFI} /$ instrumental spikes close to the expected frequency of the putative absorption, which may conceal any possible detection (Table 1 5 This leaves 16 good spectra of 14 different targets, 11 of which have not been previously searched.

In the optically thin regime (where $\tau \lesssim 0.3$ ), the total neutral hydrogen column density is related to the velocity integrated optical depth of the H I 21-cm absorption via

$$
N_{\mathrm{HI}} \approx 1.823 \times 10^{18} \frac{T_{\mathrm{s}}}{f} \int \tau d v
$$

where $T_{\mathrm{s}}$ is the spin temperature of the gas, which is a measure of the excitation from the lower hyperfine level (Purcell \& Field 1956; Field 1959) and $\int \tau d v$ is the observed velocity integrated optical depth of the absorption. In order to compare our limits with previous surveys, all spectra are re-sampled to the same spectral resolution $\left(20 \mathrm{~km} \mathrm{~s}^{-1}\right.$, as in Fig. 1, which is used as the FWHM to obtain the integrated optical depth limit, thus giving the $N_{\mathrm{H}} f / T_{\text {spin }}$ limit per channel (see Curran 2012).

Of the 11 new sources searched, for which a limit could be obtained, there were no detections of H I 21-cm absorption. Although the limits are not as sensitive as many of the other searches, particularly those at low redshift (Fig. $\sqrt{2}$, there are 42 detections and 116 previous non-detections over the range of sensitivities searched $\left[N_{\mathrm{H}}=0.1-2.4 \times 10^{19}\left(T_{\mathrm{s}} / f\right) \mathrm{cm}^{-2}\right]$. This gives a detection rate of $27 \%$ over all redshifts within these sensitivity limits, which

\footnotetext{
5 All of the spikes arise in spectra taken with the GBT, since the single dish limits the options to mitigate the RFI. Another consequence is that the large beam at these frequencies (HPBW $\approx 40^{\prime}$ ), means that the offmeasurement is very likely to contain another radio source. This will affect the flux calibration, leading to the differences from the GMRT values for the same source (which has HPBW $\lesssim 0.5^{\prime}$ and no off-measurement), although the optical depths are expected to be unaffected Ries 2012).

${ }^{6}$ For example, at $z>2$ the most sensitive limit is $N_{\mathrm{H} \text { I }}=10^{17}\left(T_{\mathrm{S}} / f\right)$ $\mathrm{cm}^{-2}$ and at $z \leqslant 2$ there are seven searches below this limit.
}

is close to the overall detection rate $(30.5 \%$, Sect. 3.2) and comparable to the $25 \%$ found by Gupta et al. (2006). Note that the highest detection rate, of $40 \%$, was obtained by Vermeulen et al. (2003) in a survey of $z \lesssim 1$ compact radio sources, where we may expect a maximum of $\approx 50 \%$, due to the alignment between the gaseous disk and continuum source along our sight-line (see Curran \& Whiting 2010). From binomial statistics, we therefore expect $3.8 \pm 1.6$ new detections for the 14 different targets with good spectra.

\subsection{Ionising photon rates}

Following our usual procedure (e.g. Curran et al. 2013b), for each target we obtained the photometry from NASA/IPAC Extragalactic Database (NED), the Wide-Field Infrared Survey Explorer (WISE, Wright et al. 2010), Two Micron All Sky Survey (2MASS, Skrutskie et al. 2006) and the Galaxy Evolution Explorer (GALEX data release GR6/7 8 databases. Each flux, $S_{\nu}$, was corrected for Galactic extinction (Schlegel et al. 1998), before being converted to a specific luminosity at the source-frame frequency, via $L_{\nu}=$ $4 \pi D_{\mathrm{L}}^{2} S_{\nu} /(z+1)$, where $D_{\mathrm{L}}$ is the luminosity distance to the source, given by

$$
D_{\mathrm{L}}=D(z+1), \text { where } D=\frac{c}{H_{0}} \int_{0}^{z} \frac{d z}{H_{\mathrm{z}} / H_{0}}
$$

is the line-of-sight co-moving distance (e.g. Peacock 1999), in which $c$ is the speed of light, $H_{0}$ the Hubble constant, $H_{\mathrm{z}}$ the Hubble parameter at redshift $z$ and

$$
\frac{H_{\mathrm{z}}}{H_{0}}=\sqrt{\Omega_{\mathrm{m}}(z+1)^{3}+\left(1-\Omega_{\mathrm{m}}-\Omega_{\Lambda}\right)(z+1)^{2}+\Omega_{\Lambda}},
$$

where we use a standard $\Lambda$ cosmology, with $H_{0}=$ $71 \mathrm{~km} \mathrm{~s}^{-1} \mathrm{Mpc}^{-1}, \Omega_{\mathrm{m}}=0.27$ and $\Omega_{\Lambda}=0.73$. We then fit a power law to the UV rest-frame data, allowing the ionising photon rate, $Q_{\mathrm{HI}} \equiv \int_{\nu}^{\infty}\left(L_{\nu} / h \nu\right) d \nu$, to be derived from

$\int_{\nu}^{\infty} \frac{L_{\nu}}{h \nu} d \nu$, where $\log _{10} L_{\nu}=\alpha \log _{10} \nu+\mathcal{C} \Rightarrow L_{\nu}=10^{\mathcal{C}} \nu^{\alpha}$, $\alpha$ is the spectral index and $\mathcal{C}$ the intercept, which gives

$$
\frac{10^{\mathcal{C}}}{h} \int_{\nu}^{\infty} \nu^{\alpha-1} d \nu=\frac{10^{\mathcal{C}}}{\alpha h}\left[\nu^{\alpha}\right]_{\nu}^{\infty}=\frac{-10^{\mathcal{C}}}{\alpha h} \nu^{\alpha} \text { where } \alpha<0,
$$

for the ionising photon rate.

Although there was only sufficient photometry for four of the targets which also had good spectra (Fig. 3), we can add these to the previous searches (Fig. 4.9. Of the $311 z \geqslant 0.1$ sources for which limits can be obtained, 211 have sufficient photometry to determine

7 Not only do compact sources tend to have lower ultra-violet luminosities Curran \& Whiting 2010; Allison et al. 2012), but there is an anticorrelation between the optical depth of the $21-\mathrm{cm}$ absorption and the extent of the radio source (Curran et al. 2013a).

8 http://galex.stsci.edu/GR6/\#mission

9 Compiled from de Waard et al. (1985); Mirabel 1989); van Gorkom et al. (1989); Uson et al. (1991); Carilli et al. (1992, 1998, 2007); Moore et al. (1999); Peck et al. (1999, 2000); Röttgering et al. (1999); Morganti et al. (2001); Ishwara-Chandra et al. (2003); Vermeulen et al. (2003); Curran et al. (2006, 2008, 2011a b, 2013b c, 2016a, 2017); Gupta et al. (2006); Orienti et al. (2006); Kanekar et al. (2009); Emonts et al. (2010); Salter et al. (2010); Chandola et al. (2011. 2013); Allison et al. (2012, 2015); Yan et al. (2012, 2016); Geréb et al. (2015); Srianand et al. (2015); Aditya et al. 2016, 2017); Grasha et al. (2017). 

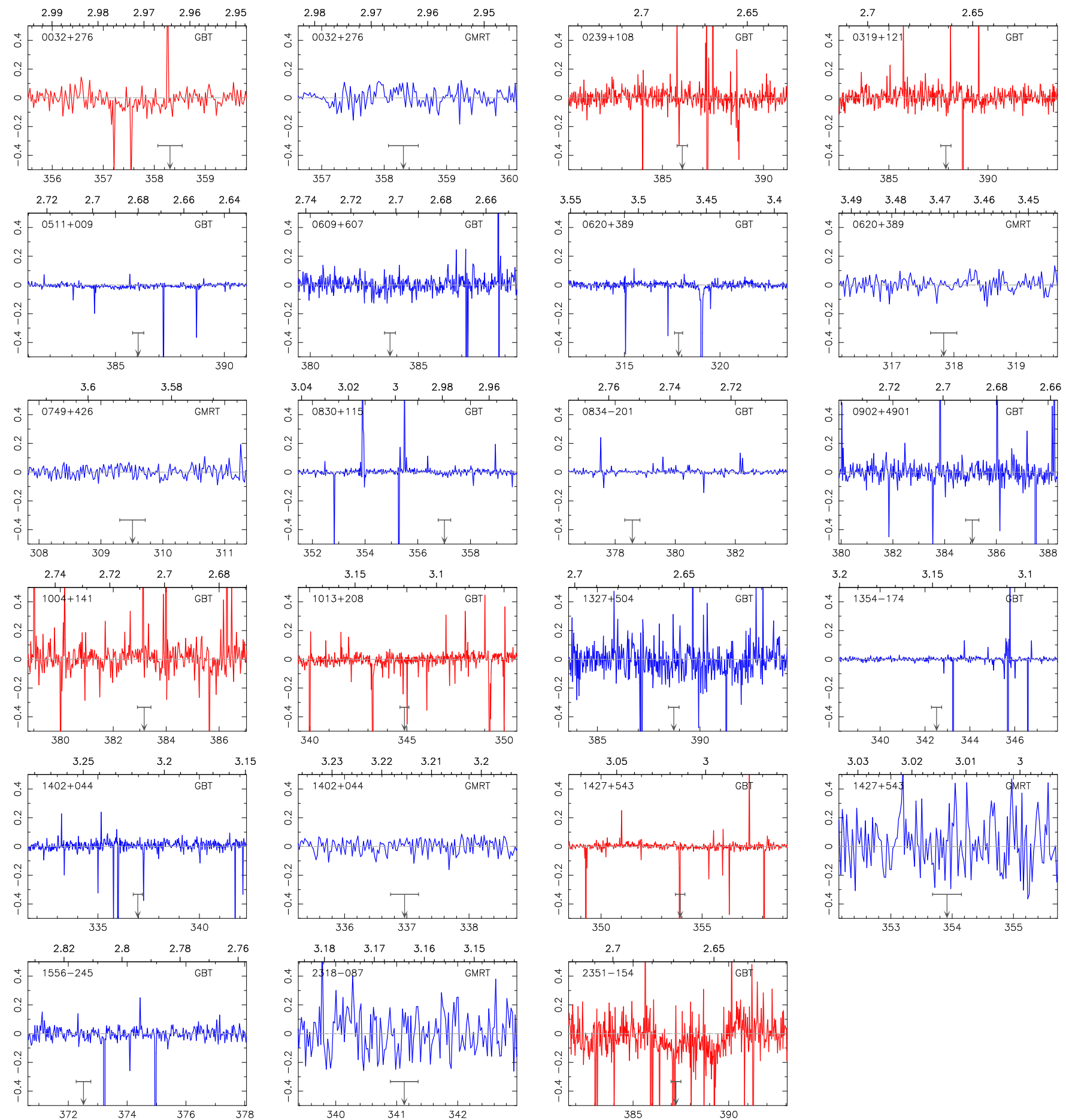

Figure 1. The reduced spectra, for which optical depth limits could be derived (Table 1 , at a spectral resolution of $20 \mathrm{~km} \mathrm{~s}^{-1}$. The ordinate gives the observed optical depth and the abscissa the barycentric frequency [MHz]. The scale along the top of each panel shows the redshift of $\mathrm{H}$ I $21-\mathrm{cm}$ over the frequency range and the downwards arrow shows the expected frequency of the absorption from the optical redshift, with the horizontal bar showing a span of $\pm 200 \mathrm{~km} \mathrm{~s}{ }^{-1}$ for guidance (the profile widths of the $21-\mathrm{cm}$ detections range from 18 to $475 \mathrm{~km} \mathrm{~s}^{-1}$, with a mean of $167 \mathrm{~km} \mathrm{~s}^{-1}$ ). The traces in red have a significant RFI spike within this range, thus potentially masking a detection. These spectra are not included in any further analysis.

the ionising photon rate. Of these, there are 43 detections and 98 non-detections with ionising photon rates up to the highest value where 21-cm absorption has been detected $\left(Q_{\mathrm{H}_{\mathrm{I}}} \leqslant 1.7 \times 10^{56} \mathrm{~s}^{-1}\right.$, Kanekar et al. 2009). Applying this $30.5 \%$ detection rate to the $Q_{\mathrm{HI}}>1.7 \times 10^{56} \mathrm{~s}^{-1}$ sources, gives a binomial probability of $8.72 \times 10^{-12}$ of obtaining 0 detections and 70 non-detections, which is significant at $6.83 \sigma$, assuming Gaussian statistics. This strengthens the case for a critical UV luminosity hindering the detection of H I 21-cm absorption at high redshift. 
Table 1. The observational results by IAU name (B1950 designation, see Table 2 for the full names as resolved by the NASA/IPAC Extragalactic Database). $z$ is the optical redshift of the source, $\Delta S$ the rms noise reached per $20 \mathrm{~km} \mathrm{~s}^{-1}$ channel, $S_{\text {meas }}$ is the measured flux density, $\tau_{3 \sigma}$ the derived optical depth limit, where $\tau_{3 \sigma}=-\ln \left(1-3 \Delta S / S_{\text {meas }}\right)$ is quoted for these non-detections. These give the quoted column densities, where $T_{\mathrm{S}}$ is the spin temperature and $f$ the covering factor; a blank field indicates that the observations were dominated by RFI or the presence of an RFI/instrumental spike close to the expected absorption redshift. The final two columns give the frequency and redshift range over which the limit applies (neglecting the spikes).

\begin{tabular}{|c|c|c|c|c|c|c|c|c|}
\hline Source & $z$ & Tel. & $\Delta S[\mathrm{mJy}]$ & $S_{\text {meas }}[\mathrm{Jy}]$ & $\tau_{3 \sigma}$ & $N_{\mathrm{HI}}\left[\mathrm{cm}^{-2}\right]$ & $\nu$-range $[\mathrm{MHz}]$ & $z$-range \\
\hline $0032+276$ & 2.9642 & GBT & 57 & 1.250 & $<0.14$ & - & $354.23-359.83$ & $2.9474-3.0099$ \\
\hline$\ldots$ & $\ldots$ & GMRT & 38 & 0.676 & $<0.17$ & $<6.1 \times 10^{18}\left(T_{\mathrm{s}} / f\right)$ & $356.50-360.33$ & $2.9420-2.9843$ \\
\hline $0239+108^{a}$ & 2.6800 & GBT & 48 & 1.032 & $<0.14$ & - & $380.48-391.06$ & $2.6322-2.7332$ \\
\hline $0319+121^{b}$ & 2.6620 & GBT & 61 & 1.510 & $<0.12$ & - & $382.51-393.83$ & $2.6067-2.7134$ \\
\hline $0511+009$ & 2.6794 & GBT & 64 & 5.208 & $<0.04$ & $<1.3 \times 10^{18}\left(T_{\mathrm{S}} / f\right)$ & $380.71-390.73$ & $2.6352-2.7309$ \\
\hline $0521-262$ & 3.109 & GMRT & - & - & - & - & \multicolumn{2}{|c|}{ RFI DOMINANT } \\
\hline $0609+607$ & 2.7020 & GBT & 54 & 1.364 & $<0.12$ & $<4.4 \times 10^{18}\left(T_{\mathrm{s}} / f\right)$ & $378.74-389.48$ & $2.6469-2.7503$ \\
\hline $0620+389^{c}$ & 3.4690 & GBT & 44 & 2.926 & $<0.05$ & $<1.6 \times 10^{18}\left(T_{\mathrm{s}} / f\right)$ & $312.11-323.60$ & $3.3894-3.5509$ \\
\hline$\ldots$ & $\ldots$ & GMRT & 11 & 0.226 & $<0.14$ & $<5.0 \times 10^{18}\left(T_{\mathrm{s}} / f\right)$ & $315.92-319.75$ & $3.4422-3.4961$ \\
\hline $0749+426^{d}$ & 3.5892 & GMRT & 19 & 0.771 & $<0.07$ & $<2.7 \times 10^{18}\left(T_{\mathrm{s}} / f\right)$ & $307.60-311.43$ & $3.5609-3.6177$ \\
\hline $0800+618^{e}$ & 3.0330 & GMRT & - & - & - & - & \multicolumn{2}{|c|}{ RFI DOMINANT } \\
\hline $0830+115$ & 2.9786 & GBT & 35 & 3.906 & $<0.04$ & $<1.3 \times 10^{18}\left(T_{\mathrm{s}} / f\right)$ & $351.37-359.90$ & $2.9467-3.0425$ \\
\hline 0834-201 & 2.7520 & GBT & 78 & 4.223 & $<0.06$ & $<2.0 \times 10^{18}\left(T_{\mathrm{s}} / f\right)$ & $375.10-384.38$ & $2.6953-2.7868$ \\
\hline $0902+490^{f}$ & 2.6887 & GBT & 60 & 0.916 & $<0.20$ & $<7.2 \times 10^{18}\left(T_{\mathrm{s}} / f\right)$ & $379.26-390.94$ & $2.6334-2.7452$ \\
\hline $0913+003$ & 3.074 & GMRT & - & - & - & - & \multicolumn{2}{|c|}{ RFI DOMINANT } \\
\hline $1004+141^{g}$ & 2.7070 & GBT & 110 & 0.614 & $<0.77^{\dagger}$ & - & $378.66-386.95$ & $2.6708-2.7511$ \\
\hline $1013+208$ & 3.1186 & GBT & 42 & 1.094 & $<0.12$ & - & $340.34-350.54$ & $3.0521-3.1735$ \\
\hline $1327+504$ & 2.6540 & GBT & 69 & 0.682 & $<0.30$ & $<1.1 \times 10^{19}\left(T_{\mathrm{s}} / f\right)$ & $383.69-394.47$ & $2.6008-2.7020$ \\
\hline $1354-174$ & 3.1470 & GBT & 41 & 3.912 & $<0.03$ & $<1.1 \times 10^{18}\left(T_{\mathrm{s}} / f\right)$ & $337.49-347.84$ & $3.0836-3.2088$ \\
\hline $1402+044$ & 3.2153 & GBT & 53 & 1.429 & $<0.11$ & $<4.1 \times 10^{18}\left(T_{\mathrm{s}} / f\right)$ & $331.44-342.91$ & $3.1422-3.2856$ \\
\hline$\ldots$ & $\ldots$ & GMRT & 17 & 0.351 & $<0.15$ & $<5.3 \times 10^{18}\left(T_{\mathrm{s}} / f\right)$ & $335.04-338.88$ & $3.1915-3.2395$ \\
\hline $1427+543$ & 3.0134 & GBT & 47 & 2.606 & $<0.05$ & - & $348.32-359.74$ & $2.9484-3.0779$ \\
\hline$\ldots$ & $\ldots$ & GMRT & 68 & 0.429 & $<0.65^{\dagger}$ & $<2.4 \times 10^{19}\left(T_{\mathrm{s}} / f\right)$ & $352.00-355.83$ & $2.9918-3.0352$ \\
\hline $1446-111$ & 2.6326 & GBT & 75 & 0.038 & - & - & $385.43-396.71$ & $2.5805-2.6852$ \\
\hline $1556-245$ & 2.8130 & GBT & 65 & 1.754 & $<0.11$ & $<4.1 \times 10^{18}\left(T_{\mathrm{s}} / f\right)$ & $369.89-378.24$ & $2.7553-2.8401$ \\
\hline $1614+051$ & 3.2150 & GMRT & 11 & 0.024 & - & - & $335.07-338.90$ & $3.1912-3.2391$ \\
\hline $1745+624$ & 3.8890 & GBT & 67 & 0.045 & - & - & $285.20-296.26$ & $3.7945-3.9805$ \\
\hline $2318-087$ & 3.1639 & GMRT & 74 & 0.505 & $<0.56^{\dagger}$ & $<2.0 \times 10^{19}\left(T_{\mathrm{s}} / f\right)$ & $339.21-343.04$ & $3.1406-3.1874$ \\
\hline $2351-154$ & 2.6680 & GBT & 81 & 0.794 & $<0.37^{\dagger}$ & - & $381.41-393.10$ & $2.6133-2.7241$ \\
\hline
\end{tabular}

Notes: ${ }^{a}$ Observed by Grasha et al. (2017) but ruined by RFI, ${ }^{b} N_{\mathrm{HI}}<6.3 \times 10^{17}\left(T_{\mathrm{S}} / f\right)$ per $30 \mathrm{~km} \mathrm{~s}^{-1}$ by Grasha et al. (2017),

${ }^{c} N_{\mathrm{H}_{\mathrm{I}}}<3.6 \times 10^{17}\left(T_{\mathrm{S}} / f\right)$ per $30 \mathrm{~km} \mathrm{~s}^{-1}$ by Aditya et al. (2016), ${ }^{d} N_{\mathrm{H}_{\mathrm{I}}}<1.4 \times 10^{18}\left(T_{\mathrm{S}} / f\right)$ per $30 \mathrm{~km} \mathrm{~s}^{-1}$ by Aditya et al. (2016),

${ }^{e} N_{\mathrm{H} \text { I }}<2.1 \times 10^{18}\left(T_{\mathrm{S}} / f\right)$ per $26 \mathrm{~km} \mathrm{~s}^{-1}$ by Aditya et al. (2016), ${ }^{f} N_{\mathrm{H}_{\mathrm{I}}}<1.5 \times 10^{18}\left(T_{\mathrm{S}} / f\right)$ per $30 \mathrm{~km} \mathrm{~s}^{-1}$ by Grasha et al. (2017), $1004+141,{ }^{g} N_{\mathrm{HI}}<1.5 \times 10^{18}\left(T_{\mathrm{S}} / f\right)$ per $30 \mathrm{~km} \mathrm{~s}^{-1}$ by Grasha et al. (2017). ${ }^{\dagger}$ Since $\tau \gtrsim 0.3$, the optical depth limit is derived assuming $f=1$ (see Curran et al. 2017).

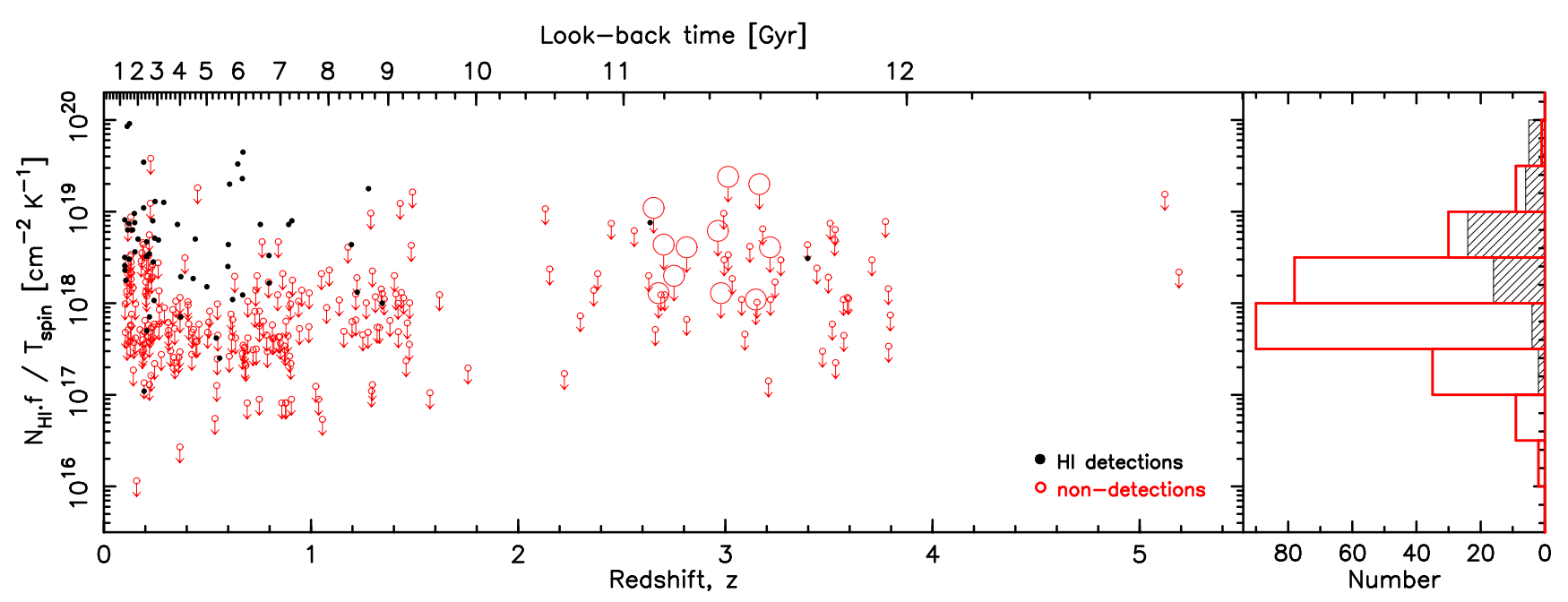

Figure 2. The line strength $\left[1.823 \times 10^{18}\left(T_{\mathrm{spin}} / f\right) \int \tau d v\right]$ versus redshift for the $z \geqslant 0.1$ associated H I 21-cm absorption searches. The filled circles/histogram represent the detections and the unfilled circles/histogram the $3 \sigma$ upper limits to the non-detections, with the large circles designating our new targets. 


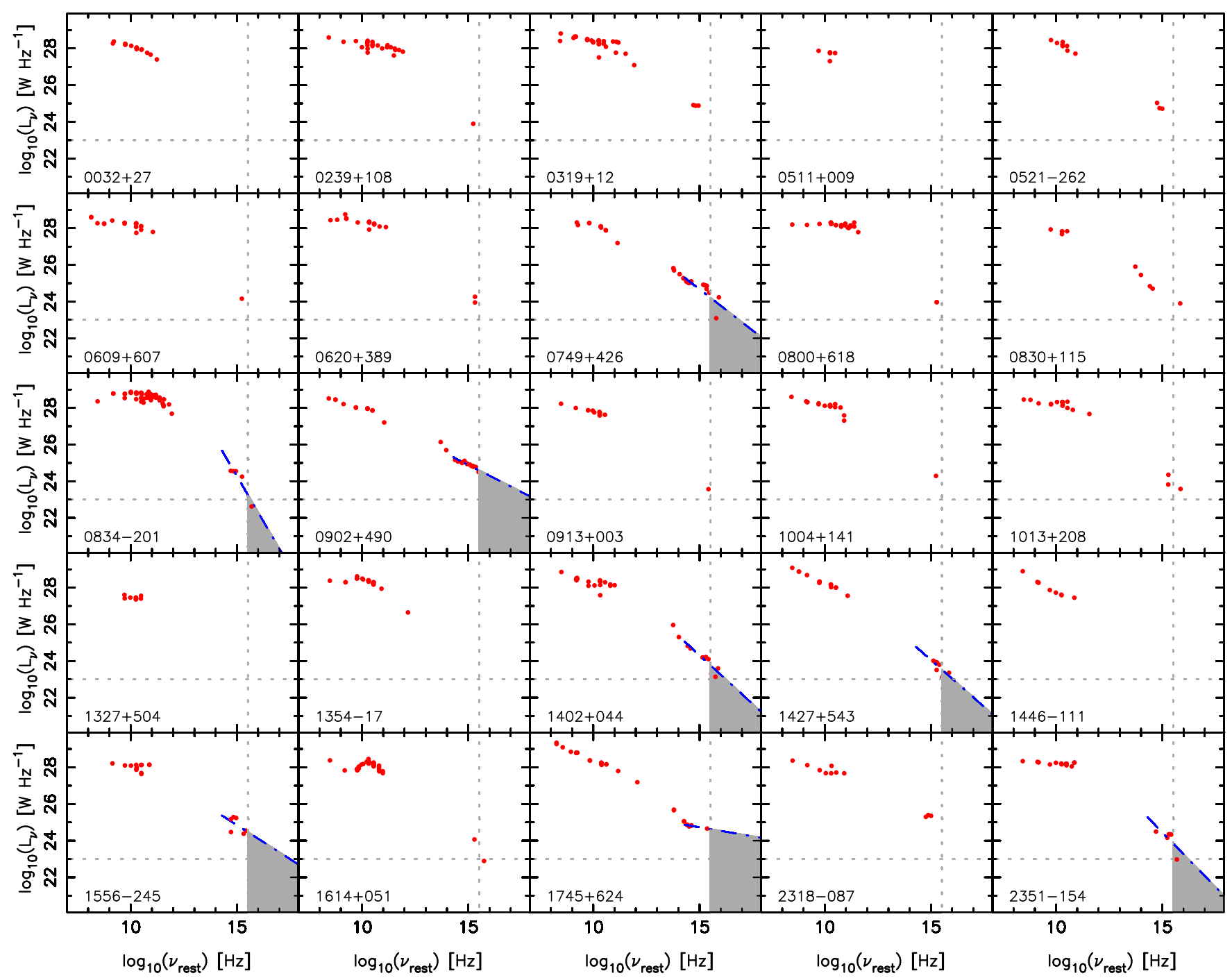

Figure 3. The rest-frame photometry for each of the 25 targets. The dashed line shows the power-law fit to the optical/UV data, the vertical dotted line signifies a rest-frame frequency of $3.29 \times 10^{15} \mathrm{~Hz}(\lambda=912 \AA)$ and the horizontal line the critical $\lambda=912 \AA$ luminosity of $L_{\mathrm{UV}} \sim 10^{23} \mathrm{~W} \mathrm{~Hz}-1$, with the shading showing the region over which the ionising photon rate is derived (Table 2 .

\section{SUMMARY}

We have undertaken a survey for associated $\mathrm{H}$ I 21 -cm absorption at redshifts of $z=2.6-3.9$ in 25 radio sources with the Green Bank and Giant Metrewave Radio Telescope, from which we obtain zero detections. Of the 14 for which limits could be obtained, 11 are new, with ten of these reaching optical depths of $\tau_{3 \sigma} \leqslant 0.3$. All of the targets were selected from the sources in the Second Realization of the International Celestial Reference Frame by Very Long Baseline Interferometry, for which 1682 have measured redshifts spanning up to $z=6.2$. Our intent was to undertake a large survey over all of this redshift space in order to quantify the incidence of associated H I 21-cm absorption over the history of the Universe. However, observing time was only awarded for the highest redshift sample, where we do not expect absorption due to all of the neutral gas being ionised in the $z \gtrsim 1$ sources that have a reliable optical redshift (Curran \& Whiting 2012).

Nevertheless, the ICRF2 provides a large new sample of strong flat-spectrum radio sources within which to search for 21$\mathrm{cm}$ absorption, and the expectation of a null result should not prevent us from testing it. Given the general $\approx 30 \%$ detection rate at the sensitivities obtained, we expect $\approx 4$ new detections, although obtaining zero detections is within $\approx 2 \sigma$ of this. In the context of all the previous searches, there still remains only two detections in this redshift range (Uson et al. 1991; Moore et al. 1999), both of which are below the ionising photon rate of $Q_{\mathrm{H}_{\mathrm{I}}} \sim 10^{56} \mathrm{~s}^{-1}$ (a monochromatic $\lambda=912 \AA$ luminosity of $L_{\mathrm{UV}} \sim 10^{23} \mathrm{~W} \mathrm{~Hz}^{-1}$ ). The addition of the five new sources for which there is sufficient photometry 10 , gives a binomial probability of $8.72 \times 10^{-12}$ of the observed distribution occuring by chance. This is significant at $6.83 \sigma$, which supports the hypothesis that $Q_{\mathrm{H}_{\mathrm{I}}} \sim 10^{56} \mathrm{~s}^{-1}$ is sufficient to ionise all of the neutral gas in a large spiral galaxy Curran \& Whiting 2012). This appears to be an ubiquitous effect, independent of source selection and applicable over all redshifts, with the selection of sources with $Q_{\mathrm{H}_{\mathrm{I}}} \lesssim 10^{56} \mathrm{~s}^{-1}$ being required to detect neutral hydrogen. This ionising photon rate corresponds to blue magnitudes of $B \gtrsim 22$ at $z \gtrsim 3$, for which there are currently no radio sources with a measured optical redshift known (Curran et al. 2013c; Morganti et al. 2015).

10 The four new plus one searched in common with Aditva et al. (2016). 


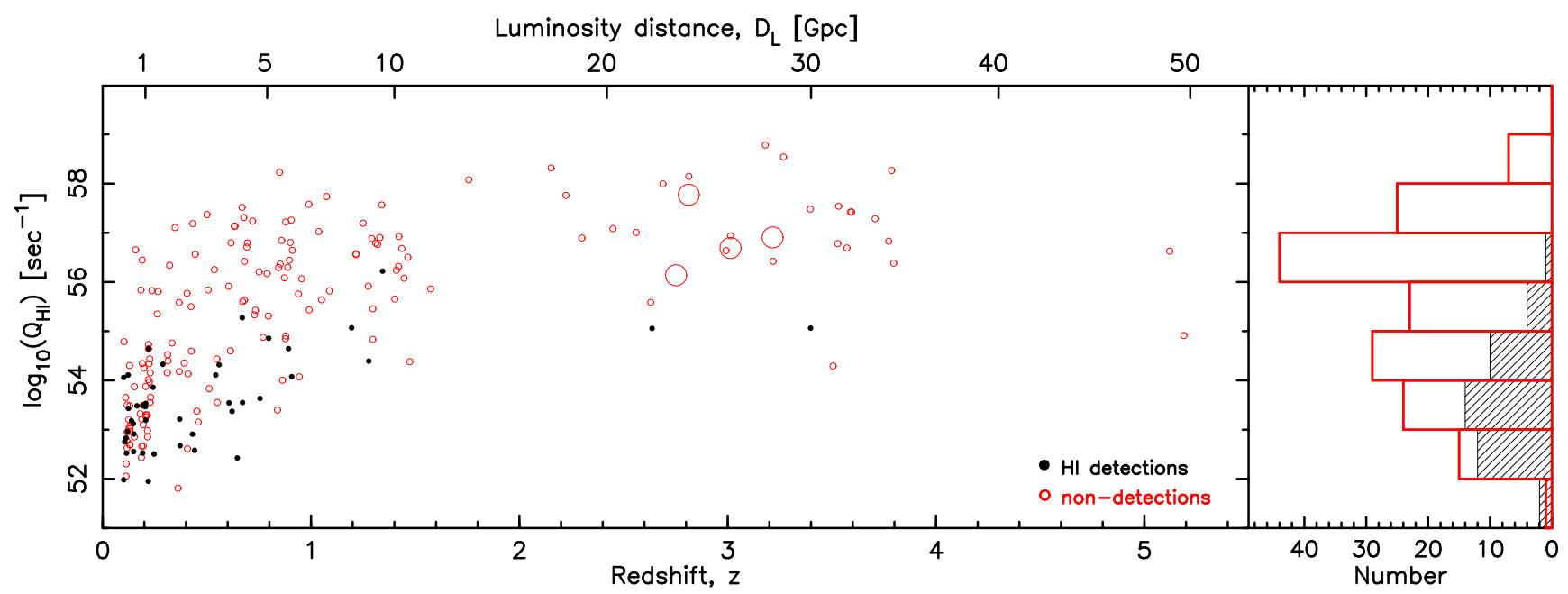

Figure 4. The ionising $(\lambda \leqslant 912 \AA)$ photon rate versus redshift for the $z \geqslant 0.1 \mathrm{H} \mathrm{I} 21-\mathrm{cm}$ absorption searches. The symbols and histogram are as per Fig. 2 where we see that the two $z>2$ detections are below the critical ionising photon rate $(4 \mathrm{C}+05.19$ at $z=2.64$, Moore et al. $1999 \& \mathrm{~B} 20902+34$ at $z=3.40$, Uson et al. 1991). Note that $0749+426$ at $z=3.5892-\log _{10} Q_{\mathrm{HI}}=57.71$ is not flagged as one of our targets since this was observed to a deeper limit by Aditya et al. (2016), Table1

Table 2. The ionising photon rates [photons s ${ }^{-1}$ ] of the targets for which a limit to the absorption strength could be obtained.

\begin{tabular}{|c|c|c|c|}
\hline NED name & IAU & $z$ & $\log _{10} Q_{\mathrm{HI}}$ \\
\hline B2 $0032+27$ & $0032+276$ & 2.9642 & - \\
\hline PKS 0239+108 & $0239+108$ & 2.680 & - \\
\hline PKS 0319+12 & $0319+121$ & 2.662 & - \\
\hline PMN J0513+0100 & $0511+009$ & 2.6770 & - \\
\hline PKS 0521-262 ${ }^{\dagger}$ & $0521-262$ & 3.109 & - \\
\hline BZQ J0614+6046 & $0609+607$ & 2.702 & - \\
\hline B2 $0620+38$ & $0620+389$ & 3.469 & - \\
\hline B3 $0749+426$ & $0749+426$ & 3.5892 & $57.71 \pm 0.78$ \\
\hline WISE J080518.15+614424. $0^{\dagger}$ & $0800+618$ & 3.033 & - \\
\hline [HB89] 0830+115 & $0830+115$ & 2.9786 & - \\
\hline [HB89] 0834-201 & 0834-201 & 2.752 & $57.52 \pm 0.12$ \\
\hline SDSS J090527.46+485049.9 & $0902+490$ & 2.6887 & $57.99 \pm 0.20$ \\
\hline SDSS J091551.69+000713.2 ${ }^{\dagger}$ & $0913+003$ & 3.074 & - \\
\hline [HB89] 1004+141 & $1004+141$ & 2.707 & - \\
\hline PKS 1014+208 & $1013+208$ & 3.1186 & - \\
\hline SDSS J132905.80+500926.5 & $1327+504$ & 2.654 & - \\
\hline PKS 1354-17 & 1354-174 & 3.147 & - \\
\hline [HB89] 1402+044 & $1402+044$ & 3.2153 & $57.04 \pm 0.47$ \\
\hline SDSS J142921.87+540611.1 & $1427+543$ & 3.0134 & $56.69 \pm 0.51$ \\
\hline PKS B1446-111 & $1446-111$ & 2.6326 & - \\
\hline [HB89] 1556-245 & $1556-245$ & 2.813 & $57.77 \pm 0.78$ \\
\hline [HB89] 1614+051 ${ }^{\dagger}$ & $1614+051$ & 3.2150 & - \\
\hline $4 \mathrm{C}+62.29^{\dagger}$ & $1745+624$ & 3.889 & $58.51 \pm 0.07$ \\
\hline PKS 2318-087 & $2318-087$ & 3.1639 & - \\
\hline [HB89] 2351-154 & $2351-154$ & 2.668 & $56.94 \pm 0.90$ \\
\hline
\end{tabular}

${ }^{\dagger}$ Shown for completeness as no sensitivity limit could be derived.

We therefore reiterate that the detection of neutral gas within the hosts of high redshift active galaxies requires us to dispense with the usual reliance upon an optical redshift to which to define the search frequency. The way forward is through wide-band observations of radio sources of unknown redshift with the Square Kilometre Array.

\section{ACKNOWLEDGEMENTS}

We wish to thank the anonymous referee for their helpful comments, as well as Katie Grasha and Jeremy Darling for a draft manuscript of their forthcoming paper. We also thank the staff of the GMRT who have made these observations possible. GMRT is run by the National Centre for Radio Astrophysics of the Tata Institute of Fundamental Research. The National Radio Astronomy Observatory is a facility of the National Science Foundation operated under cooperative agreement by Associated Universities, Inc This research has made use of the NASA/IPAC Extragalactic Database (NED) which is operated by the Jet Propulsion Laboratory, California Institute of Technology, under contract with the National Aeronautics and Space Administration. This research has also made use of NASA's Astrophysics Data System Bibliographic Services.

\section{REFERENCES}

Aditya J. N. H. S., Kanekar K., Prochaska J. X., Day B., Lynam P., Cruz J., 2017, MNRAS, 465, 5011

Aditya J. N. H. S., Kanekar N., Kurapati S., 2016, MNRAS, 455, 4000

Allison J. R. et al., 2012, MNRAS, 423, 2601

Allison J. R. et al., 2015, MNRAS, 453, 1249

Begeman K. G., Broeils A. H., Sanders R. H., 1991, MNRAS, 249,523

Carilli C. L., Menten K. M., Reid M. J., Rupen M. P., Yun M. S., 1998, ApJ, 494, 175

Carilli C. L., Perlman E. S., Stocke J. T., 1992, ApJ, 400, L13

Carilli C. L., Wang R., van Hoven M. B., Dwarakanath K., Chengalur J. N., Wyithe S., 2007, AJ, 133, 2841

Chandola Y., Gupta N., Saikia D. J., 2013, MNRAS, 429, 2380

Chandola Y., Sirothia S. K., Saikia D. J., 2011, MNRAS, 418, 1787

Curran S. J., 2012, ApJ, 748, L18

Curran S. J., 2017, MNRAS, in press (arXiv:1704.04294)

Curran S. J., Allison J. R., Glowacki M., Whiting M. T., Sadler E. M., 2013a, MNRAS, 431, 3408

Curran S. J., Allison J. R., Whiting M. T., Sadler E. M., Combes 
F., Pracy M. B., Bignell C., Athreya R., 2016a, MNRAS, 457, 3666

Curran S. J., Duchesne S. W., Divoli A., Allison J. R., 2016b, MNRAS, 462, 4197

Curran S. J., Murphy M. T., Pihlström Y. M., Webb J. K., Purcell C. R., 2005, MNRAS, 356, 1509

Curran S. J., Webb J. K., 2006, MNRAS, 371, 356

Curran S. J., Whiting M. T., 2010, ApJ, 712, 303

Curran S. J., Whiting M. T., 2012, ApJ, 759, 117

Curran S. J., Whiting M. T., Allison J. R., Tanna A., Sadler E. M., Athreya R., 2017, MNRAS, 467, 4514

Curran S. J. et al., 2011a, MNRAS, 413, 1165

Curran S. J., Whiting M. T., Murphy M. T., Webb J. K., Longmore S. N., Pihlström Y. M., Athreya R., Blake C., 2006, MNRAS, 371,431

Curran S. J., Whiting M. T., Sadler E. M., Bignell C., 2013b, MNRAS, 428, 2053

Curran S. J., Whiting M. T., Tanna A., Sadler E. M., Pracy M. B., Athreya R., 2013c, MNRAS, 429, 3402

Curran S. J., Whiting M. T., Webb J. K., Athreya R., 2011b, MNRAS, 414, L26

Curran S. J., Whiting M. T., Wiklind T., Webb J. K., Murphy M. T., Purcell C. R., 2008, MNRAS, 391, 765

Davis M. M., May L. S., 1978, ApJ, 219, 1

de Waard G. J., Strom R. G., Miley G. K., 1985, A\&A, 145, 479

Emonts B. H. C. et al., 2010, MNRAS, 406, 987

Field G. B., 1959, ApJ, 129, 536

Geréb K., Maccagni F. M., Morganti R., Oosterloo T. A., 2015, A\&A, 575, 44

Grasha K., Darling J., 2011, in American Astronomical Society Meeting Abstracts, Vol. 43, p. 345.02

Grasha K., Darling J. K., Bolatto, A. D. Leroy A., Stocke J., 2017, ApJ, submitted

Gupta N., Salter C. J., Saikia D. J., Ghosh T., Jeyakumar S., 2006, MNRAS, 373, 972

Ishwara-Chandra C. H., Dwarakanath K. S., Anantharamaiah K. R., 2003, JA\&A, 24, 37

Kalberla P. M. W., Dedes L., Kerp J., Haud U., 2007, A\&A, 469, 511

Kanekar N., Chengalur J. N., 2003, A\&A, 399, 857

Kanekar N., Prochaska J. X., Ellison S. L., Chengalur J. N., 2009, MNRAS, 396, 385

Kanekar N. et al., 2014, MNRAS, 438, 2131

Ma C. et al., 2009, IERS Technical Note, 35, 1

Maccagni F. M., Morganti R., Oosterloo T. A., Geréb K., Maddox N., 2017, A\&A, in press (arXiv:1705.00492)

Mirabel I. F., 1989, ApJ, 340, L13

Moore C. B., Carilli C. L., Menten K. M., 1999, ApJ, 510, L87

Morganti R., Oosterloo T. A., Tadhunter C. N., van Moorsel G., Killeen N., Wills K. A., 2001, MNRAS, 323, 331

Morganti R., Sadler E. M., Curran S., 2015, Advancing Astrophysics with the Square Kilometre Array (AASKA14), 134

Noterdaeme P. et al., 2012, A\&A, 547, L1

Orienti M., Morganti R., Dallacasa D., 2006, A\&A, 457, 531

Osterbrock D. E., 1989, Astrophysics of Gaseous Nebulae and Active Galactic Nuclei. University Science Books, Mill Valley, California

Peacock J. A., 1999, Cosmological Physics. Cambridge University Press, Cambridge

Peck A. B., Taylor G. B., Conway J. E., 1999, ApJ, 521, 103

Peck A. B., Taylor G. B., Fassnacht C. D., Readhead A. C. S., Vermeulen R. C., 2000, ApJ, 534, 104
Purcell E. M., Field G. B., 1956, ApJ, 124, 542

Rao S., Turnshek D., Nestor D. B., 2006, ApJ, 636, 610

Ries P. A., 2012, PhD thesis, University of Virginia

Röttgering H., de Bruyn G., Pentericci L., Miley G., 1999, in The

Most Distant Radio Galaxies, Röttgering H. J. A., Best P. N., Lehnert M. D., eds., p. 113

Salter C. J., Saikia D. J., Minchin R., Ghosh T., Chandola Y., 2010, ApJ, 715, L117

Schlegel D. J., Finkbeiner D. P., Davis M., 1998, ApJ, 500, 525

Skrutskie M. F. et al., 2006, AJ, 131, 1163

Srianand R., Gupta N., Momjian E., Vivek M., 2015, MNRAS, 451, 917

Titov O., Malkin Z., 2009, A\&A, 506, 1477

Titov O., Stanford L. M., Johnston H. M., Pursimo T., Hunstead R. W., Jauncey D. L., Maslennikov K., Boldycheva A., 2013, AJ, 146,10

Uson J. M., Bagri D. S., Cornwell T. J., 1991, PhRvL, 67, 3328

van Gorkom J. H., Knapp G. R., Ekers R. D., Ekers D. D., Laing R. A., Polk K. S., 1989, AJ, 97, 708

Vermeulen R. C. et al., 2003, A\&A, 404, 861

Wright E. L. et al., 2010, AJ, 140, 1868

Yan T., Stocke J. T., Darling J., Hearty F., 2012, AJ, 144, 124

Yan T., Stocke J. T., Darling J., Momjian E., Sharma S., Kanekar N., 2016, AJ, 151, 74 